Research Article

\title{
Risk Factors for Moderate to Severe Pain during the First 24 Hours after Laparoscopic Bariatric Surgery While Receiving Intravenous Patient-Controlled Analgesia
}

\author{
Arissara Iamaroon, Suwimon Tangwiwat, Patchareya Nivatpumin, Thidarat Lertwacha, \\ Piyawadee Rungmongkolsab $\mathbb{D}$, and Pawinee Pangthipampai $\mathbb{D}$
}

Department of Anesthesiology, Faculty of Medicine, Siriraj Hospital, Mahidol University, Bangkok, Thailand

Correspondence should be addressed to Pawinee Pangthipampai; pawinee141@gmail.com

Received 23 February 2019; Revised 25 June 2019; Accepted 9 August 2019; Published 3 October 2019

Academic Editor: Basavana B. Goudra

Copyright (c) 2019 Arissara Iamaroon et al. This is an open access article distributed under the Creative Commons Attribution License, which permits unrestricted use, distribution, and reproduction in any medium, provided the original work is properly cited.

\begin{abstract}
Objective. To investigate the incidence of and risk factors for moderate to severe pain during the first 24 hours after laparoscopic bariatric surgery. Materials and Methods. This retrospective study included morbidly obese patients who underwent laparoscopic sleeve gastrectomy or Roux-en-Y gastric bypass at a single institution between June 2016 and July 2018. Demographic, clinical, operative, and postoperative pain data from the postanesthesia care unit (PACU) and ward were analyzed. Intravenous patientcontrolled analgesia (IV-PCA) was commenced before PACU discharge. Results. Ninety-seven patients were included. The mean age was $38.60 \pm 12.27$ years, and the mean BMI was $45.04 \pm 8.42 \mathrm{~kg} / \mathrm{m}^{2}$, and $69 \%$ were female. The incidence of moderate to severe pain was $75 \%$. Moderate to severe pain during the first 24 hours was associated with young age, female sex, postoperative administration of NSAIDs, first pain score greater than 3 on arrival at the PACU, and inadequate pain control at PACU discharge. A multivariate analysis revealed that inadequate pain control at PACU discharge was the only factor independently associated with moderate to severe pain during the first 24 hours postoperatively $(p=0.011)$. From PACU discharge to the end of postoperative day 3, moderate to severe pain at the end of each 24-hour period was a significant predictor of moderate to severe pain in the subsequent 24-hour period ( $p=0.011, p<0.001$, and $p=0.004$, respectively). Conclusions. Moderate to severe pain was experienced by $75 \%$ of patients undergoing laparoscopic bariatric surgery and receiving IV-PCA after PACU discharge. Inadequate pain control at PACU discharge was the only independent risk factor for moderate to severe pain during the first 24 hours postoperatively.
\end{abstract}

\section{Introduction}

Pain management is challenging in morbidly obese patients [1]. As the first-line drug type for postoperative pain control in these patients is opioids, there is a substantial risk of opioidinduced ventilatory impairment (OIVI) [2]. Moreover, the morbidly obese population has a high prevalence of obstructive sleep apnea (OSA) [3, 4], and the optimal opioid dosages in morbidly obese patients with OSA versus without OSA is not yet known [5]. Concerns about prescribing opioids in morbidly obese patients can lead to inadequate postoperative pain control and resultant adverse consequences.
Intravenous (IV) patient-controlled analgesia (PCA), a pain management strategy that allows patients to titrate their own opioid demand within the analgesic corridor, minimizes the incidence of side effects like OIVI [6, 7]. IV-PCA may be a suitable strategy for postoperative pain control in morbidly obese patients after discharge from the post-anesthesia care unit (PACU). However, to the best of our knowledge, no studies have investigated the efficacy of IVPCA for controlling pain after laparoscopic bariatric surgery in morbidly obese patients.

The present study aimed to investigate the incidence of and risk factors for moderate to severe pain during the first 
24 hours postoperatively in morbidly obese patients who had undergone laparoscopic bariatric surgery and received postoperative IV-PCA before discharge from the PACU.

\section{Materials and Methods}

This retrospective study included morbidly obese patients who underwent either laparoscopic sleeve gastrectomy or Roux-en-Y gastric bypass at a single, large, tertiary referral center between June 2016 and July 2018. Patients were excluded if IV-PCA was not started postoperatively, or if the data relating to their pain scores or the administered analgesics were missing. The study protocol was approved by the Institutional Review Board of our institution.

Records from both the ward staff and the acute pain service were reviewed. The following data were collected: age; sex; BMI; American Society of Anesthesiologists classification; the presence and severity of OSA, diagnosed from the results of a sleep test as mild (Apnea-Hypopnea Index $(\mathrm{AHI}) \geq 5$, but $<15$ ), moderate (AHI5, but $<30$ ), or severe $(\mathrm{AHI} \geq 30)$; the presence of continuous positive airway pressure use in patients with OSA; and tobacco use (nonsmoking indicated that the patient had stopped using tobacco at least 30 days preoperatively). The surgical data collected were: type of laparoscopic bariatric surgery (Rouxen-Y gastric bypass or sleeve gastrectomy), concomitant surgical procedures, and operative time.

As to information from the PACU, the pain scores at the patients' arrival and discharge and the details of all analgesics given were collected. All patients achieved at least 9 out of a maximum possible score of 10 on our hospital postanesthetic recovery scoring system before discharge from PACU. The scoring elements were movement ( 0 , no movement; 1, purposeless movement; 2, purposeful movement); respiration ( 0 , apnea; 1 , dyspnea or limited; 2 , deep breath and cough); hemodynamics (0, blood pressure change more than $50 \%$ from preoperative baseline; 1 , $20 \%-50 \%$ change from baseline; 2 , change within $20 \%$ baseline); consciousness ( 0 , not responding; 1 , arousable on calling; 2 , alert and oriented); and color (0, cyanotic; 1 , pale, dusky, and jaundiced; 2, normal).

The pain scores and details of all analgesic drugs administered in the period between the PACU discharge and 1 day after discontinuation of the PCA were obtained from ward staff and acute pain service records. Pain severity was rated using a 0-10 numeric rating scale (NRS): 0 indicated no pain, 1-3 mild pain, 4-6 moderate pain, 7-10 severe pain, and 10 maximal pain. Inadequate pain control was defined as moderate to severe pain on the previous day; as the present study focused on the first 24 hours postoperatively, inadequate pain control was defined as moderate to severe pain in the PACU. Nursing ward staff recorded the patient pain score every 4 hours, while the acute pain service recorded the pain score both at rest and during movement once a day or during a follow-up check after adjusting the analgesic regimen. The moderate-to-severe pain group comprised patients that had an NRS $>3$ at any assessment time during the first 24 hours postoperatively.
All morbidly obese patients who underwent laparoscopic bariatric surgery were prescribed IV-PCA with the setting of bolus morphine $1 \mathrm{mg}$, a lockout of $5 \mathrm{~min}$, and a $4 \mathrm{~h}$-maximum dose of $30 \mathrm{mg}$. The daily PCA demand, delivery counts, and cumulative dose were recorded. The dosages of all opioid analgesics were converted to IV morphine equivalents (IV morphine $10 \mathrm{mg}$ is equal to IV fentanyl $100 \mathrm{mcg}$ and equal to IV pethidine $75 \mathrm{mg}$ ) [8]. A demand-to-delivery ratio greater than 1.35 was considered a measure of the quality of analgesia, and a more objective method of analgesic assessment than verbal methods of assessment [9]. The severity and management of all complications were analyzed. Sedation was rated as 0 (none), 1 (mild, occasionally drowsy, and easy to arouse), 2 (moderate, constantly or frequently drowsy, and easy to arouse), 3 (severe, somnolent, and difficult to arouse), or $S$ (normal sleep and easy to arouse). Respiratory depression was defined as a respiratory rate lower than 8 breaths/min, and it was rated as absent or present. Nausea, vomiting, and pruritus were rated as 0 (none), 1 (mild, requiring no treatment), 2 (moderate with resolution via medication), or 3 (severe and persistent despite medication). The administration of NSAIDs was recorded; they comprised ketorolac, parecoxib, ibuprofen, and celecoxib. Pre- and intraoperative multimodal anesthesia beyond local anesthetic wound infiltration (which was given in all cases) was recorded, including premedication with analgesic drugs, intraoperative ketamine, and any regional anesthesia.

2.1. Sample Size Calculation and Statistical Analysis. The sample size was calculated based on a previously reported incidence of $70 \%$ for moderate to severe pain in patients who had undergone laparoscopic bariatric surgery [10]. Using a two-sided test with a 95\% confidence interval and an allowable error of 0.1 , the calculated sample size was 81 patients. That figure was increased by $20 \%$ in 100 patients to compensate for excluded cases.

All statistical analyses were performed using PASW Statistics for Windows, version 18.0 (SPSS Inc., Chicago, IL, USA). Continuous data are presented as the mean \pm standard deviation, while categorical data are shown as number and percentage. Data were tested for normal distribution using the Kolmogorov-Smirnov test. As to the univariate analysis, the association of the categorical variables with moderate to severe pain was assessed by the chi-squared test, while the association of the continuous variables with moderate to severe pain was assessed by Student's $t$-test. Variables with a $p$ value $<0.20$ in the univariate analysis were included in the multivariate analysis. A $p$ value $<0.05$ was regarded as statistically significant.

\section{Results}

Ninety-seven patients were included. The mean age was $38.60 \pm 12.27$ years, and the mean BMI was $45.04 \pm 8.42 \mathrm{~kg} / \mathrm{m} 2$, and $69 \%$ were female. The incidences of moderate to severe pain drawn from the records of the acute pain service and the ward staff are presented in Table 1. 
TABLe 1: Pain scores during the first 24 hours after laparoscopic bariatric surgery $(n=97)$.

\begin{tabular}{lcc}
\hline Pain data & No or mild pain (NRS 0-3) & Moderate to severe pain (NRS 4-10) \\
\hline Recorded by the acute pain service & & \\
Pain at rest & $65(67.0 \%)$ & $32(33.0 \%)$ \\
Pain during movement & $23(23.7 \%)$ & $74(76.3 \%)$ \\
Recorded by the ward nursing staff & $25(25.8 \%)$ & $72(74.2 \%)$ \\
\hline
\end{tabular}

Values are given as $n$ (\%). Abbreviation: NRS, numeric rating scale.

TABLe 2: Demographic and surgical data related to pain severity.

\begin{tabular}{|c|c|c|c|}
\hline Data & No or mild pain (NRS $0-3 ; n=25$ ) & Moderate to severe pain (NRS $4-10 ; n=72$ ) & $p$ value \\
\hline Age (years) & $41.60 \pm 12.34$ & $37.56 \pm 12.16$ & 0.157 \\
\hline Female sex & $14(56.0 \%)$ & $53(73.6 \%)$ & 0.101 \\
\hline BMI $\left(\mathrm{kg} / \mathrm{m}^{2}\right)$ & $43.56 \pm 6.14$ & $45.55 \pm 9.06$ & 0.311 \\
\hline ASA classification & & & 0.613 \\
\hline 2 & $5(20.0 \%)$ & $18(25.0 \%)$ & \\
\hline 3 & $20(80.0 \%)$ & $54(75.0 \%)$ & \\
\hline Obstructive sleep apnea & & & 0.796 \\
\hline None & $12(48.0 \%)$ & $37(51.4 \%)$ & \\
\hline Mild & $2(8.0 \%)$ & $6(8.3 \%)$ & \\
\hline Moderate & $2(8.0 \%)$ & $9(12.5 \%)$ & \\
\hline Severe & $9(9.0 \%)$ & $20(27.8 \%)$ & \\
\hline CPAP (yes) & $12(48.0 \%)$ & $31(43.1 \%)$ & 0.668 \\
\hline Type of operation & & & 0.514 \\
\hline Roux-en-Y & $12(48.0 \%)$ & $40(55.6 \%)$ & \\
\hline Sleeve gastrectomy & $13(52.0 \%)$ & $32(44.4 \%)$ & \\
\hline Concomitant operations & & & 0.586 \\
\hline Hiatal hernia & $1(4.0 \%)$ & $1(1.4 \%)$ & \\
\hline $\mathrm{LC}$ & $3(12.0 \%)$ & $5(6.9 \%)$ & \\
\hline LC and hiatal hernia & $0(0.0 \%)$ & $2(2.8 \%)$ & \\
\hline Operative time (min) & $171.92 \pm 67.03$ & $178.29 \pm 66.25$ & 0.680 \\
\hline
\end{tabular}

$p<0.05$ indicates statistical significance. Values are given as $n$ (\%) or mean \pm standard deviation. Abbreviations: NRS, numeric rating scale; BMI, body mass index; ASA, American Society of Anesthesiologists; CPAP, continuous positive airway pressure; LC, laparoscopic cholecystectomy.

TABle 3: Pain and analgesic data during intraoperative, PACU, and postoperative periods.

\begin{tabular}{|c|c|c|c|}
\hline Data & No or mild pain (NRS $0-3 ; n=25$ ) & Moderate to severe pain (NRS $4-10 ; n=72$ ) & $p$ value \\
\hline \multicolumn{4}{|l|}{ Intraoperative } \\
\hline Intraoperative morphine (mg) & $19.56 \pm 5.39$ & $20.62 \pm 6.43$ & 0.463 \\
\hline Intraoperative NSAIDs & $4(16 \%)$ & $9(12.5 \%)$ & 0.735 \\
\hline Multimodal analgesia & $7(28.0 \%)$ & $27(37.5 \%)$ & 0.391 \\
\hline \multicolumn{4}{|l|}{$P A C U$} \\
\hline NRS $>3$ on arrival & $14(56 \%)$ & $55(76.4 \%)$ & 0.053 \\
\hline PACU morphine (mg) & $1.18 \pm 1.80$ & $1.69 \pm 2.22$ & 0.305 \\
\hline PACU NSAIDs & $1(4 \%)$ & $8(11.1 \%)$ & 0.439 \\
\hline Inadequate pain control at PACU discharge & $4(16.0 \%)$ & $32(44.4 \%)$ & 0.011 \\
\hline \multicolumn{4}{|l|}{ Postoperative } \\
\hline Demand-to-delivery ratio & $2.08 \pm 1.79$ & $1.89 \pm 0.97$ & 0.523 \\
\hline Demand-to-delivery ratio $>1.35$ & $14(56.0 \%)$ & $45(62.5 \%)$ & 0.566 \\
\hline Postoperative NSAIDs & $4(16.0 \%)$ & $24(33.3 \%)$ & 0.099 \\
\hline
\end{tabular}

$p<0.05$ indicates statistical significance. Values are given as $n(\%)$ or mean \pm standard deviation. Abbreviations: PACU, postanesthesia care unit; NRS, numeric rating scale; NSAIDs, nonsteroidal anti-inflammatory drugs.

There were no significant differences in the demographic and surgical data of the moderate-to-severe pain group versus the no-or-mild pain group (Table 2). There tended to be more females in the moderate-to-severe pain group than in the no-or-mild pain group; however, this difference was not statistically significant. The mean operative time for all patients was $176.65 \pm 66.16$ minutes.
Data relating to the patients' pain scores and analgesic drugs during the intraoperative, PACU, and postoperative periods are detailed in Table 3 . The median pain scores on arrival were 4 (range $0-10$ ) for the no-or-mild pain group vs $7(0-10)$ for the moderate-to-severe pain group $(p=0.038)$. By comparison, the median pain scores at discharge were 3 $(0-6)$ vs $3(0-7)$, respectively $(p=0.103)$. The multimodal 
TABLE 4: Multivariate analysis of factors associated with moderate to severe pain during the first 24 hours postoperatively.

\begin{tabular}{lcr}
\hline Factors & Adjusted OR (95\% CI) & $p$ value \\
\hline Age & $0.96(0.92-1.01)$ & 0.104 \\
Female sex & $2.68(0.91-7.91)$ & 0.075 \\
Postoperative NSAIDs & $3.29(0.91-11.85)$ & 0.069 \\
Patients with NRS $>3$ on arrival at PACU & $1.03(0.31-3.46)$ & 0.961 \\
Inadequate pain control at PACU discharge & $4.90(1.44-16.69)$ & $\mathbf{0 . 0 1 1}$ \\
\hline
\end{tabular}

$p<0.05$ indicates statistical significance. Abbreviations: OR, odds ratio; CI, confidence interval; NSAIDs, nonsteroidal anti-inflammatory drugs.

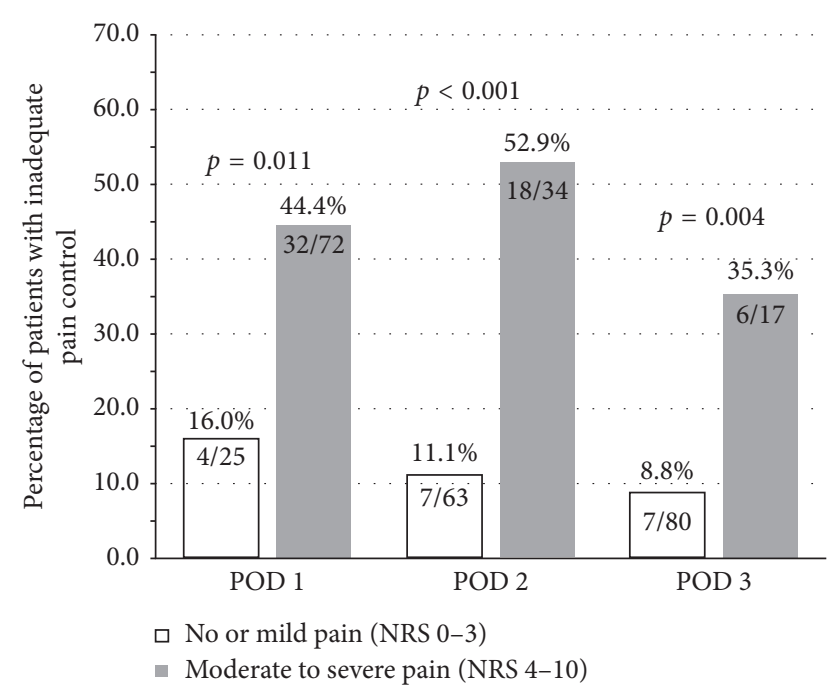

Figure 1: Percentage of patients with inadequate pain control at the end of the previous 24-hour period on postoperative days 1-3. $p<0.05$ indicates statistical significance. Abbreviations: POD, postoperative day; NRS, numeric rating scale.

data for the pre- and intraoperative periods revealed that two patients $(20.6 \%)$ received a premedication (one received $1 \mathrm{~g}$ of paracetamol, while the other received $1 \mathrm{~g}$ of paracetamol plus $400 \mathrm{mg}$ of ibuprofen); all patients had wound infiltration with local anesthesia, using $20 \mathrm{ml}$ of $0.5 \%$ bupivacaine; one patient received $50 \mathrm{mg}$ of ketamine intraoperatively; and three patients received a bilateral quadratus lumborum block. Only inadequate pain control at PACU discharge was significantly more frequent for the moderate-to-severe pain group than for the no-or-mild pain group. Postoperative NSAID usage tended to be more common in the moderate-to-severe pain group, but the intergroup difference was not significant. The amount of morphine required during the first 24 hours was $20.60 \pm 15.77$ vs $29.17 \pm 18.86 \mathrm{mg}(p=0.44)$ for the no-ormild pain and the moderate-to-severe pain groups, respectively.

The univariate analysis revealed that the two groups significantly differed regarding age, female sex, postoperative NSAID usage, patients with NRS $>3$ on arrival at the PACU, and inadequate pain control at PACU discharge $(p<0.2$; Tables 2 and 3$)$. When those factors were included in the multivariate analysis, only inadequate pain control at PACU discharge was identified as an independent risk factor for moderate to severe pain during the first 24 hours postoperatively (Table 4).
As to the effect of inadequate pain control at the end of the previous day on the pain status of the following day, there were significant differences between the two groups at PACU discharge $(p=0.011)$, at the end of the first 24 hours postoperatively $(p<0.001)$, and at 48 hours postoperatively $(p=0.004$; Figure 1$)$.

The adverse effects and complications resulting from IVPCA opioids did not differ between the two groups (Table 5). No patient experienced respiratory depression. The lengths of hospital stay were also similar for the groups.

\section{Discussion}

The present study revealed a high incidence $(74.2 \%)$ of moderate to severe pain in morbidly obese patients after laparoscopic bariatric surgery. In 1997, it was proposed that less than $5 \%$ of patients should experience pain after surgery by $2002^{11}$; this proclamation was intended to encourage a worldwide increase in the standard of patient care [11]. We used the pain scores from ward staff records rather than from the acute pain service records to divide the study patients into the two groups for analysis, given that the ward nurses perform pain assessments every 4 hours rather than once daily. However, the pain scores from the ward staff records were similar to the pain scores during movement from the acute pain service data. We believe that the pain score during movement during the postoperative period is important because adequate pain control during patient movement results in earlier ambulation and better postoperative outcomes [12].

Several studies have reported that younger age and female sex increase the risk of postoperative pain [13-16]; however, other studies have reported contrary findings $[17,18]$. In the present study, the multivariate analysis revealed no significant differences in age or sex between those with moderate to severe pain versus patients with no to mild pain postoperatively. More patients received NSAIDs in the moderate-to-severe pain group than in the no-or-mild pain group, which seems to contradict the fact that NSAIDs are used in multimodal analgesic protocols. However, as there was a concern that NSAID administration may cause postoperative gastric leakage and marginal ulcers, NSAIDs were only prescribed for patients with uncontrollable pain. Although NSAID usage was significantly associated with moderate to severe pain in the univariate analysis, this factor did not remain significant in the multivariate analysis.

Multimodal analgesia combines more than one type of analgesic to achieve pain control, while simultaneously reducing opioid consumption and opioid-related side effects. 
TABLE 5: Sedation scores, adverse effects, and lengths of hospital stay of the two groups.

\begin{tabular}{|c|c|c|c|}
\hline Data & No or mild pain (NRS $0-3 ; n=25$ ) & Moderate to severe pain (NRS $4-10 ; n=72$ ) & $p$ value \\
\hline Sedation score & & & 0.601 \\
\hline 0 & $23(92.0 \%)$ & $69(95.8 \%)$ & \\
\hline 1 & $2(8.0 \%)$ & $3(4.2 \%)$ & \\
\hline 2 & $0(0.0 \%)$ & $0(0.0 \%)$ & \\
\hline Respiratory depression & $0(0.0 \%)$ & $0(0.0 \%)$ & \\
\hline PONV & & & 0.654 \\
\hline 0 & $18(72.0 \%)$ & $56(77.8 \%)$ & \\
\hline 1 & $7(28.0 \%)$ & $15(20.8 \%)$ & \\
\hline 2 & $0(0.0 \%)$ & $1(1.4 \%)$ & \\
\hline Pruritus & & & 0.320 \\
\hline 0 & $24(96 \%)$ & $64(88.9 \%)$ & \\
\hline 1 & $0(0.0 \%)$ & $6(8.3 \%)$ & \\
\hline 2 & $1(4.0 \%)$ & $2(2.8 \%)$ & \\
\hline LOS & $5.08 \pm 1.15$ & $5.14 \pm 1.04$ & 0.813 \\
\hline
\end{tabular}

$p<0.05$ indicates statistical significance. Values are given as $n(\%)$ or mean \pm standard deviation. Abbreviations: PONV, postoperative nausea and vomiting; LOS, length of hospital stay.

Multimodal analgesia regimens are the core of Enhanced Recovery after Surgery protocols [19]. In the current study, only $38.1 \%$ of the patients received a multimodal analgesia; however, multimodal strategies should be used throughout the perioperative period, i.e., pre-, intra-, and postoperatively. Although the benefits of multimodal treatment are well recognized, the use of multimodal anesthesia has been limited [20]. Multimodal analgesic protocols should be strictly implemented as a mandatory hospital policy to reduce opioid use, reduce opioid-related side effects, and improve pain relief in morbidly obese patients [21].

The multivariate analysis revealed that the only independent risk factor for pain after laparoscopic gastric bypass surgery was inadequate pain control upon PACU discharge. In our study, inadequate pain control was defined as an NRS $>3$ during the previous day [22]. IV-PCA is now more commonly used in demand mode only with no background infusion [18], since the routine addition of a continuous infusion has no beneficial effects for average opioid-naive patients. More specifically, the addition of a background infusion does not always reduce the number of demands made by a patient, nor does it result in better analgesia and improved sleep patterns. Moreover, it may increase the total amount of opioid delivered, and it significantly increases the risk of OIVI [23]. The principle of using PCA in demand mode only is that patient comfort must be accomplished before the commencement of PCA. A loading dose of opioid is given until a reported pain score of 3 out of 10 is achieved before PCA is started [24] because this helps achieve the minimum effective analgesic opioid concentration. This principle helps to explain why inadequate pain control at PACU discharge was a strong predictor of moderate to severe pain within the 24 hours after PACU discharge [1]. This is despite obese patients being more sensitive to opioids and requiring much lower opioid doses to achieve similar analgesic endpoints. This awareness may lead to suboptimal treatment by virtue of inter individual variations in opioid requirements. Therefore, an individual titration would be the best way to manage pain, especially with challenging obese patients. This narrow, therapeutically effective opioid dose could be administered safely in the PACU with close monitoring and an adequate number of caregivers. The present results also revealed that inadequate pain control in the previous period (PACU discharge, 24 hours postoperatively, or 48 hours postoperatively) resulted in moderate to severe pain during the subsequent period.

IV-PCA is not suitable for all patients. Optimal pain control requires the tailoring of treatment to the individual. The optimal bolus dose is that which results in good pain relief with minimal adverse effects. Therefore, the initial dose may need to be adjusted so that IV-PCA meets the requirements of each individual patient. IV-PCA is not a "one size fits all" or "set and forget" therapy [25]. We also evaluated the demand-to-delivery ratios of the moderate-tosevere pain group and the no-or-mild pain group as this ratio is often used as an indicator of pain and the quality of analgesia [9]. The demand-to-delivery ratio points out a high number of unsuccessful demands which may reflect the need for more opioid. However, our results revealed no significant difference in the groups' ratios and more than half of the patients in each group had a demand-to-delivery ratio $>1.35$. This may be explained by patient anxiety, patient confusion, and/or inappropriate patient use of IV-PCA.

Although we found no significant differences in the postoperative complications of the groups, inadequate postoperative pain control has previously been associated with immune deficiency, slower wound healing, and the development of chronic pain [10]. Moreover, there have been reports of psychological effects, such as a reduced quality of life, sleep-related problems, and feelings of demoralization and anxiety [26, 27]. Unrelieved pain has also been found to be significantly associated with higher economic costs [27].

\section{Limitations}

This study has some limitations. Firstly, this was a retrospective study that analyzed data from a single center. Furthermore, we had no information as to whether patients 
were trained in how to correctly use IV-PCA. Future research should attempt to identify barriers to the effective management of acute pain, and the consequences of inadequate pain management in morbidly obese patients.

\section{Conclusions}

The incidence of moderate to severe pain in patients who underwent laparoscopic bariatric surgery and received IVPCA after PACU discharge was $75 \%$. Inadequate pain control at discharge from the PACU was the only independent risk factor for moderate to severe pain during the first 24 hours postoperatively. Caregivers should improve pain management in this patient population to improve the level of patient care.

\section{Data Availability}

The data from this clinical study will be available in SPSS format for sharing provided all conditions stipulated by the local university are met.

\section{Ethical Approval}

All procedures performed in studies involving human participants were in accordance with the ethical standards of the institutional and/or national research committee and with the 1964 Helsinki Declaration and its later amendments or comparable ethical standards.

\section{Conflicts of Interest}

All authors declare that they do not have any personal or professional conflicts of interest related to this study.

\section{Acknowledgments}

This study was funded by a grant from the Faculty of Medicine, Siriraj Hospital, Mahidol University, Bangkok, Thailand (grant no. R016131062 [Fund 3]). The authors gratefully acknowledge Nichapat Thongkaew of the Department of Anesthesiology for her assistance with data collection and Julaporn Pooliam of the Division of Clinical Epidemiology, Department of Research and Development, Faculty of Medicine, Siriraj Hospital, for her assistance with the statistical analysis.

\section{References}

[1] A. Alvarez, P. M. Singh, and A. C. Sinha, "Postoperative analgesia in morbid obesity," Obesity Surgery, vol. 24, no. 4, pp. 652-659, 2014.

[2] S. A. Chung, H. Yuan, and F. Chung, "A systemic review of obstructive sleep apnea and its implications for anesthesiologists," Anesthesia and Analgesia, vol. 107, no. 5, pp. 1543$1563,2008$.

[3] W. C. Frey and J. Pilcher, "Obstructive sleep-related breathing disorders in patients evaluated for bariatric surgery," Obesity Surgery, vol. 13, no. 5, pp. 676-683, 2003.

[4] P. P. Lopez, B. Stefan, C. I. Schulman, and P. M. Byers, "Prevalence of sleep apnea in morbidly obese patients who presented for weight loss surgery evaluation: more evidence for routine screening for obstructive sleep apnea before weight loss surgery," The American Surgeon, vol. 74, no. 9, pp. 834-838, 2008.

[5] K. K. Lam, S. Kunder, J. Wong, A. G. Doufas, and F. Chung, "Obstructive sleep apnea, pain, and opioids: is the riddle solved?," Current Opinion in Anesthesiology, vol. 29, no. 1, pp. 134-140, 2016.

[6] S. J. Dolin, J. N. Cashman, and J. M. Bland, "Effectiveness of acute postoperative pain management: I. Evidence from published data," British Journal of Anaesthesia, vol. 89, no. 3, pp. 409-423, 2002.

[7] J. Hudcova, E. McNicol, C. Quah, J. Lau, and D. B. Carr, "Patient controlled opioid analgesia versus conventional opioid analgesia for postoperative pain," Cochrane Database Syst Rev, vol. 4, Article ID CD003348, 2006.

[8] R. Payne, M. Max, A. Sunshine, C. Inturrisi, A. Rogers, and A. Miser, "Principles of analgesic use in the treatment of acute pain and chronic cancer pain, 2nd edition, American Pain Society," Clinical Pharmacy, vol. 9, no. 8, pp. 601-612, 1990.

[9] E. P. McCoy, G. Furness, and P. M. Wright, "Patient-controlled analgesia with and without background infusion. Analgesia assessed using the demand: delivery ratio," $A n$ aesthesia, vol. 48, no. 3, pp. 256-260, 1993.

[10] M. Hartwig, R. Allvin, R. Backstrom, and E. Stenberg, "Factors associated with increased experience of postoperative pain after laparoscopic gastric bypass surgery," Obesity Surgery, vol. 27, no. 7, pp. 1854-1858, 2017.

[11] B. Fitzsimons, L. Wilton, T. Lamont, L. McCulloch, and J. Boyce, "The audit commission review of diabetes services in england and wales, 1998-2001," Diabetic Medicine, vol. 19, no. s4, pp. 73-78, 2002.

[12] O. A. Bamgbade, O. Oluwole, and R. R. Khaw, "Perioperative analgesia for fast-track laparoscopic bariatric surgery," Obesity Surgery, vol. 27, no. 7, pp. 1828-1834, 2017.

[13] H. J. Gerbershagen, E. Pogatzki-Zahn, S. Aduckathil et al., "Procedure-specific risk factor analysis for the development of severe postoperative pain," Anesthesiology, vol. 120, no. 5, pp. 1237-1245, 2014.

[14] J. Thomazeau, A. Rouquette, V. Martinez et al., "Acute pain Factors predictive of post-operative pain and opioid requirement in multimodal analgesia following knee replacement," European Journal of Pain, vol. 20, no. 5, pp. 822-832, 2016.

[15] M. S. Cepeda and D. B. Carr, "Women experience more pain and require more morphine than men to achieve a similar degree of analgesia," Anesthesia and Analgesia, vol. 97, no. 5, pp. 1464-1468, 2003.

[16] B. Kest, E. Sarton, and A. Dahan, "Gender differences in opioid-mediated analgesia: animal and human studies," Anesthesiology, vol. 93, no. 2, pp. 539-547, 2000.

[17] A. Zeidan, S. Al-Temyatt, H. Mowafi, and T. Ghattas, "Gender-related difference in postoperative pain after laparoscopic Roux-En-Y gastric bypass in morbidly obese patients," Obesity Surgery, vol. 23, no. 11, pp. 1880-1884, 2013.

[18] C. W. Cheung, C. L. Ying, L. H. Lee, S. F. Tsang, S. L. Tsui, and M. G. Irwin, "An audit of postoperative intravenous patientcontrolled analgesia with morphine: evolution over the last decade," European Journal of Pain, vol. 13, no. 5, pp. 464-471, 2009.

[19] M. Tan, L. S. Law, and T. J. Gan, "Optimizing pain management to facilitate Enhanced Recovery after Surgery pathways," Canadian Journal of Anesthesia, vol. 62, no. 2, pp. 203-218, 2015. 
[20] R. D. Urman, E. A. Boing, V. Khangulov et al., "Analysis of predictors of opioid-free analgesia for management of acute post-surgical pain in the United States," Current Medical Research and Opinion, vol. 35, no. 2, pp. 283-289, 2019.

[21] J. J. Ng, W. Q. Leong, C. S. Tan et al., “A multimodal analgesic protocol reduces opioid-related adverse events and improves patient outcomes in laparoscopic sleeve gastrectomy," Obesity Surgery, vol. 27, no. 12, pp. 3075-3081, 2017.

[22] H. J. Gerbershagen, J. Rothaug, C. J. Kalkman, and W. Meissner, "Determination of moderate-to-severe postoperative pain on the numeric rating scale: a cut-off point analysis applying four different methods," British Journal of Anaesthesia, vol. 107, no. 4, pp. 619-626, 2011.

[23] P. E. Macintyre, "Safety and efficacy of patient-controlled analgesia," British Journal of Anaesthesia, vol. 87, no. 1, pp. 36-46, 2001.

[24] K. Gandhi, J. L. Baratta, J. W. Heitz, E. S. Schwenk, B. Vaghari, and E. R. Viscusi, "Acute pain management in the postanesthesia care unit," Anesthesiology Clinics, vol. 30, no. 3, pp. e1-e15, 2012.

[25] R. C. Etches, "Patient-controlled analgesia," Surgical Clinics of North America, vol. 79, no. 2, pp. 297-312, 1999.

[26] T. J. Gan, "Poorly controlled postoperative pain: prevalence, consequences, and prevention," Journal of Pain Research, vol. 10, pp. 2287-2298, 2017.

[27] R. Sinatra, "Causes and consequences of inadequate management of acute pain," Pain Medicine, vol. 11, no. 12, pp. 1859-1871, 2010. 


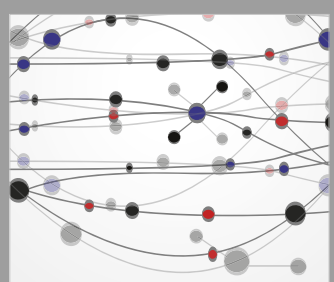

The Scientific World Journal
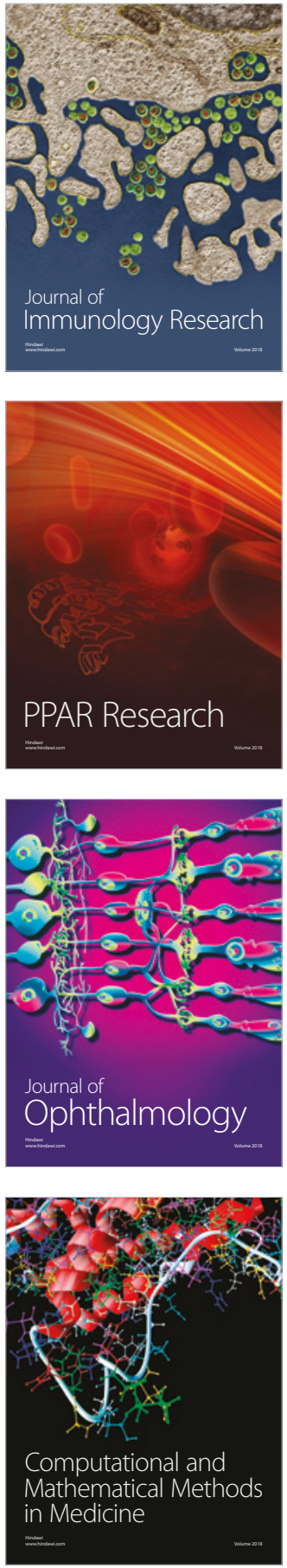

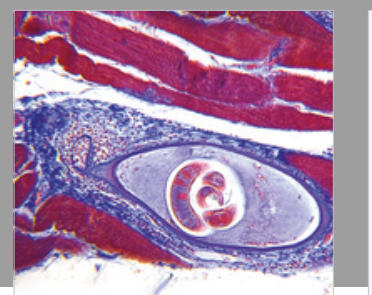

Gastroenterology Research and Practice

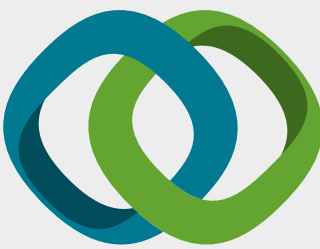

\section{Hindawi}

Submit your manuscripts at

www.hindawi.com
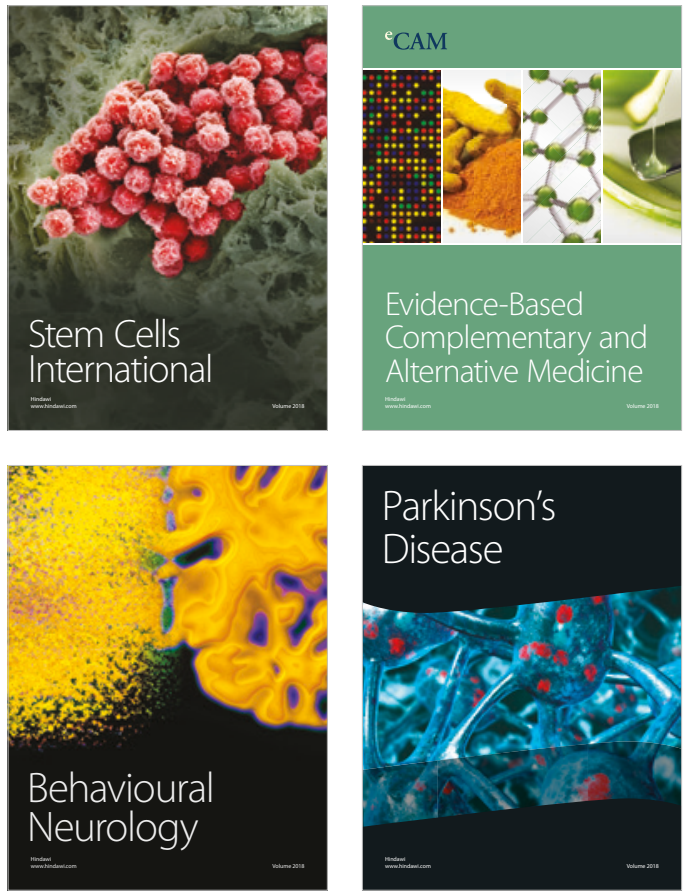

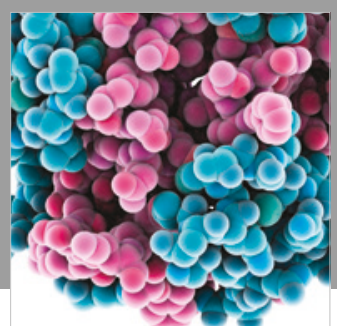

ournal of

Diabetes Research

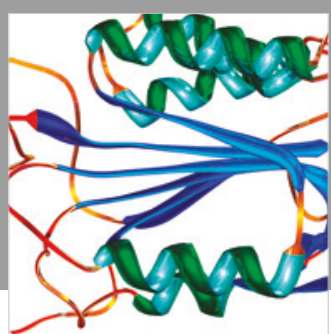

Disease Markers
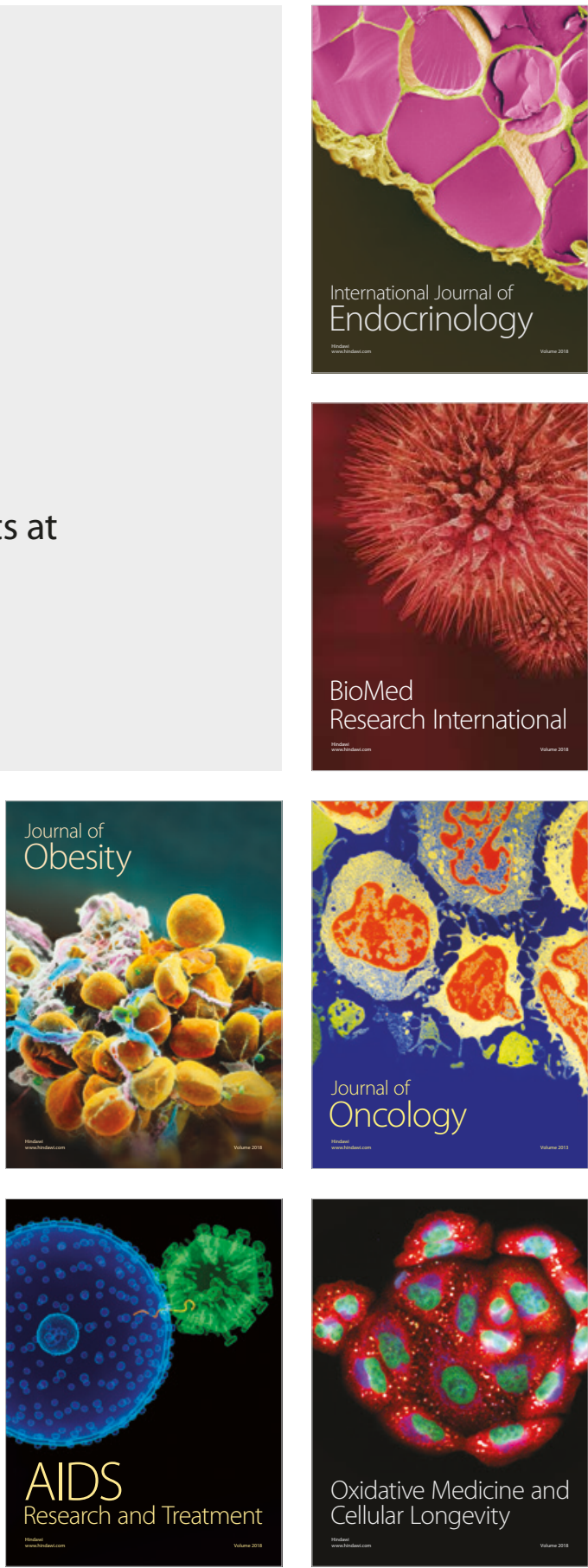